pathogenesis of some of these conditions. In the section on slow viruses, it would have perhaps been useful to have discussed scrapie and its pathogenesis in more detail and also to have covered Visna infection of sheep.

Overall the authors have done a fine job and have produced a thoroughly worthwhile volume which is extremely well referenced. The book is not cheap, but in view of its length the price is not unreasonable. I shall certainly find this useful as a ready to hand reference source and $I$ am sure that it deserves to be on the shelves of any good neurological library.

PGE KENNEDY

\section{Companion to Psychiatric Studies 4th edition.} Edited by R E Kendell, A K Zeally. (Pp 827; £42.50.) Edinburgh: Churchill Livingstone, 1988.

The Companion to Psychiatric Studies has for many years been the principal textbook used by postgraduate students of psychiatry, though its ascendant position has been challenged recently by the appearance of two competing textbooks from the Maudsley Hospital and Oxford. The fourth addition of the book will without doubt re-establish its position as the leader in the field.

The problems confronting the editors of any textbook of psychiatry are enormous. The subject does not have the clear boundaries as, for instance, a discipline like neurology. Knowledge of the causes of the major mental illnesses is still in its infancy, and for many conditions treated by psychiatrists there are competing and often mutually exclusive perspectives. This leads to the difficulty of how to be comprehensive without being over inclusive. The principal North American textbook of psychiatry covers three weighty volumes and exemplifies the latter problem. The Companion, in marked contrast, manages in less than $\mathbf{8 0 0}$ pages to give a clear and concise overview of the subject.

In this new edition the editors have introduced two completely new chapters, on psychiatric epidemiology and epilepsy, as well as re-writes, by new authors, of seven other chapters. All the remaining chapters have been substantially updated. The format of the book is as in previous editions, with the early sections focusing on the sciences and allied disciplines relevant to psychiatry. The remaining 28 chapters cover the range of problems seen in psychiatric practice including sections on mental handicap, child psychiatry, adolescent psychiatry and psychiatric disorders of child birth. Professor Kendell contributes all three chapters on the major psychoses and it would be hard to find a better summary of the current state of knowledge.

No text book can claim to be totally comprehensive and its aims should be more modest, serving as a source of basic information, as well as providing a jumping-off point for further research and study. In this respect the Companion to Psychiatric Studies will remain a valuable guide to postgraduate students of psychiatry, psychiatrists and other professionals seeking an accessible and sound guide to the field.

RJ DOLAN

Applied Neurophysiology. By J A Simpson, W Fitch. (Pp 358; £35.00.) Guildford: Butterworth Scientific, 1988.

This is a text book of human physiology directed specifically towards anaesthetists, who as the authors note, probably now comprise the largest hospital speciality. The authors' aim has been to provide a description of the general principles and crucial concepts necessary for a sound understanding of nervous system function. Coverage is comprehensive but with emphasis on such areas of particular relevance to anaesthetists as pain, respiration and mechanism of arousal, attention and consciousness.

The book comprises 28 chapters arranged in five groups. Part 1 is entitled Cellular Organisation of the Nervous System and is followed by The Afferent Nervous System, Higher Nervous Functions, Interaction with the Environment and finally Regulation of the Internal Environment. In general the text is very clearly written and much more readable than is often the case with works in this field. The title is perhaps somewhat misleading since the clinical content is rather less than I for one had anticipated. For example, although the physiology of the neuromuscular function is of course well described, there is surprisingly little on myasthenia and related disorders. The authors make a particular point of the illustrations, noting that in many publications there is over simplification, and especially omission of the connections of the mid-brain and diencephalon. Here an attempt has been made to give a truer representation but this results in some exceedingly complicated figures, not wholly remedied by the unusually lengthy figure legends. I wonder how much the addition of colour would have added to the cost of the book - shades of grey are really inadequate for figures of this complexity.

This work will be a most useful addition to the library of any department of anaesthethetics or neurology and is competitively priced to be affordable by the individual; those who do buy it and who make use of it will consider the money well spent.

NMP MURRAY

Cranial Magnetic Resonance Imaging. By Allen D Elster. (Pp 420; £75.00.) Edinburgh: Churchill Livingstone 1988.

Since 1980 magnetic resonance imaging (MRI) has evolved from the research laboratory to become a routine part of diagnostic radiology and although there is a plethora of specialist journal articles and several books devoted to it, the overall place of MRI in the diagnosis of cranial disease is only now being established. Allen Elster in his latest text from the Bowman Gray School of Medicine, North Carolina, has attempted to consolidate the sum of knowledge of cranial MRI and condense this information into an easily assimilated form which he claims is more than just a book on "CT with different pictures". How far has he succeeded in explaining these concepts of biophysics and imaging strategies?

Generally, his aims are fulfilled: the book is divided into twelve chapters starting with the now obligatory descriptions of MRI machines and the physical principles of the signals produced. This is followed by a comprehensive explanation of pulse sequences with good examples of the different appearances when each parameter in turn is changed. These early sections are marred only by the incorrect underestimated values given for $\mathrm{T} 1$ and $\mathrm{T} 2$ of liquids such as CSF or urine which should have relaxation times of at least $3,000 \mathrm{~ms}$ and $2,500 \mathrm{~ms}$ respectively (pages 13, 15). This introduction leads to sections on normal anatomy, normal variants and congenital and developmental anomalies. They adequately cover the common diagnostic areas.

In the following chapters cerebral disease is described on a novel patho-anatomical basis being divided into a discussion of the ventricles and subarachnoid spaces in health and disease, neoplasms, cerebral vascular disease, infections and inflammatory diseases, degenerative diseases and abnormalities of the skull base and orbit. Chapter 10 entitled "Hemorrhage, trauma and 